\title{
Investigación-acción y didáctica de las lenguas: del posicionamiento del investigador a una postura de investigación
}

Dominique Macaire*

* Laboratorio ATILF, Université de Lorraine, Francia.

Correo electrónico:

dominique.macaire@univ-lorraine.fr

Recibido: 5 de junio del 2015

Aprobado: 5 de agosto del 2015

Cómo citar este artículo: Macaire, Dominique. "Investigación-acción y didáctica de las lenguas: del posicionamiento del investigador a una postura de investigación". Trads. Juan Felipe Zuluaga Molina y Alejandro Arroyave Tobón. Rastros Rostros 17.31 (2015): 157-165. Impreso. doi: http://dx.doi. org/10.16925/ra.v17i31.1097

\section{Resumen}

La investigación en didáctica de las lenguas y las culturas encuentra su lugar como campo de investigación en la medida en que sea reconocida en todo el sentido de las palabras que la nombran, algo que está lejos de ocurrir en el pensamiento científico contemporáneo. De hecho, la didáctica de las lenguas sufre de un déficit de credibilidad, lo cual puede constatarse en su dificultad para posicionarse en el seno mismo de las disciplinas. Además, la didáctica de las lenguas no se asume como una ciencia, sino como un campo que toma recursos de lo humano y del aprendiz de lenguas, generalmente en un contexto institucional. En fin, vive de sus relaciones con ciencias conexas como la sociolingüística o la psicolingüística, por ejemplo, de la cual se nutre y a la cual enriquece a la vez. La DDLC trata las adquisiciones y las compara con los aprendizajes de las lenguas en ambientes diversos y, aunque estos campos sean herméticos, permiten al individuo apropiaciones diversas de un patrimonio plurilingüe y pluricultural.

Palabras clave: didáctica de las lenguas y culturas, investigación-acción, metodología de investigación. 


\title{
Action Research and Language Teaching: From the Positioning of the Researcher to a Research Position
}

\begin{abstract}
Research in didactics of languages and cultures finds its place as a field of research to the extent that it is recognized in every sense of the words that name it, something that is far from occurring in contemporary scientific thought. In fact, the didactics of languages suffers from a credibility deficit, which can be seen in its difficulty to position in the bosom of disciplines. In addition, the didactics of languages is not assumed as a science, but as a field that takes resources from the human and the language learner, usually in an institutional context. In short, it lives by its relationships with related sciences such as sociolinguistics or psycholinguistics, which nourishes didactics and is enriched by it. The didactics of languages and cultures (DDLC) deals with acquisitions and compares them with the learning of languages in different environments and, although these areas are hermetic, allow various appropriations by the individual of a multilingual and multicultural heritage.
\end{abstract}

Keywords: didactics of languages and cultures, action research, research methodology.

\section{Investigação-ação e didática das línguas: do posicionamento do investigador a uma posição de investigação}

\section{Resumo}

A investigação em didática das línguas e as culturas encontra o seu lugar como campo de investigação na medida em que seja reconhecida em todo o sentido das palavras que a nomeiam, uma questão que está longe de acontecer no pensamento científico contemporâneo. De fato, didática das línguas padece um déficit de credibilidade, o que se pode verificar na sua dificuldade para se posicionar no seio próprio das disciplinas. Além disso, a didática das línguas não se assume como uma ciência, mas como um campo que se emprega dos recursos do humano e do aprendiz de línguas, geralmente em um contexto institucional. A fim, vive das suas relações com ciências correlatas como a sociolinguística ou a psicolinguística, por exemplo, da qual se nutre e à qual por sua vez enriquece. A didática das línguas e as culturas (DDLC) trata das aquisições e são comparadas com os aprendizados das línguas em ambientes diversos y, embora estes campos sejam herméticos, permitem ao indivíduo diversas apropriações de um patrimônio plurilíngue e pluricultural.

Palavras-chave: didática das línguas e culturas, investigação-ação, metodologia de investigação. 


\section{Introducción ${ }^{1}$}

Una de las metodologías de investigación privilegiada por la didáctica de las lenguas y las culturas (en adelante, DDLC) es la investigación-acción (en adelante, IA). Ni investigación fundamental, ni investigación aplicada, la IA es cambiante e indeterminada. Más aún, acepta esta incómoda apertura y es justamente por esto que este término puede ser incomprendido o desvalorizado con respecto a la investigación científica, la cual es determinada. De acuerdo con Hess:

La recherche-action peut se définir comme un processus collectif mettant en relation des chercheurs et des praticiens visant à produire un savoir en prise directe sur les pratiques des acteurs sociaux. Cette définition très large étant posée, elle permet ensuite toutes les variations. Aujourd'hui, la recherche-action dans le champ éducatif est extrêmement variée. (9) ${ }^{2}$

Heredada de Dewey, luego desarrollada por Lewin, la IA nació en la década del cuarenta en Estados Unidos y se expandió en la posguerra y la reconstrucción (ver acerca de este tema Thirion; Goyette y Lessard-Hebert). Su origen, implicado en el marco social alrededor de la dinámica de grupos, es aún hoy complicado en sus modalidades de implementación en ciencias humanas y sociales. Es asimismo campo de intervención en problemas generados en grupos constituidos. En la lógica de la IA, los investigadores están implicados y pueden actuar al lado de otros actores en un dispositivo. Esta proximidad de la acción confiere una fuente de saberes para la investigación en términos de ayuda al cambio.

\footnotetext{
1 El presente artículo apareció originalmente en la publicación Les Après-midi de LAIRDIL en 2010. La referencia específica es: Macaire, Dominique. "Recherche-action et didactique des langues: du positionnement du chercheur à une posture de recherche". Les Après-midi de LAIRDIL, 17 (2010): 21-32. Puede ser consultado en línea en https://hal.archives-ouvertes.fr/hal-00554831/document. Traductores: Juan Felipe Zuluaga Molina y Alejandro Arroyave Tobón. Correos electrónicos: juanf.zuluaga@udea.edu.co; alejandro.arroyave@udea.edu.co.Traductores del Grupo de Investigación TNT (Traducción y Nuevas Tecnologías de la Escuela de Idiomas de la Universidad de Antioquia.

2 Nuestra traducción: "La investigación-acción puede definirse como un proceso colectivo que relaciona a los investigadores y a los practicantes con el objetivo de producir un saber en relación directa con las prácticas de los actores sociales. Esta definición bastante amplia y ya establecida, permite luego cualquier variación. Actualmente, la investigación-acción en el campo educativo es extremadamente variada." (9).
}

Tal como lo expone Montagne-Macaire:

La volonté de résoudre des problèmes rencontrés dans la société américaine s’adosse à l’idée que les acteurs des communautés eux-mêmes peuvent prendre appui sur leurs propres actions en tant que groupe, et produire du changement d'attitudes et de comportements. $(99)^{3}$

En relación con la DDLC, Jean-Paul NarcyCombes presenta la IA en estos términos:

L'objet qu'étudie la didactique des langues, même s'il semble plus pertinent de parler de L2, est une pratique sociale, et dont la recherche-action se révèle être la méthodologie de recherche la plus adaptée à cet objet. $(7)^{4}$

Podríamos entonces preguntarnos de qué naturaleza son las intervenciones llevadas a cabo por las investigaciones implicadas, y qué ambigüedad reside en estas metodologías. El término mismo de intervención no es del todo fiable en el campo de las ciencias exactas, en la medida en que permite suponer una ausencia de distancia crítica y de objetividad por parte del investigador.

\section{La investigación-acción, un proceso dinámico}

En estrecha relación con las teorías de la acción, la iA en DDLC se caracteriza por tres elementos dinámicos: proceso(s), procesador(es) y producto(s) (Macaire, Monisme ou pluralisme?). Se encuentran el resto de estos elementos en las descripciones de los investigadores de la IA social (Bazin; Barbier).

En términos de proceso, la IA se articula alrededor de tres metodologías principales: la observación, la intervención y la etnografía escolar, con la investigación-formación particularmente. Estas no son impermeables entre ellas, sino que mantienen

\footnotetext{
$3 \quad$ Nuestra traducción: "La voluntad para resolver problemas encontrados en la sociedad estadounidense se soporta en la idea de que los actores de las comunidades pueden apoyarse en sus propias acciones como grupo y producir un cambio en las actitudes y comportamientos" (Montagne-Macaire, 99).

$4 \quad$ Nuestra traducción: "El objeto que estudia la didáctica de las lenguas, aun cuando parezca más pertinente hablar de L2, es una práctica social, y es donde la investigación-acción parece ser la metodología de investigación más adaptada a este objeto" (NarcyCombes 7).
} 
contratos de relaciones. Lo que interesa a la investigación aquí tiene que ver con la descripción y la interpretación de los datos y con la intención de la acción sobre estos... De hecho, en una lógica de acción el proceso es central (Habermas). Es entonces en los vínculos e interrelaciones donde se encuentra el meollo de la investigación.

En términos de producto y de intención, la iA en DDLC permite conocer mejor las prácticas por su descripción $y$, a veces, actuar sobre ellas de forma incitativa o prescriptiva, en beneficio de las instituciones o de los responsables.

En cuanto a los procesadores, los actores de la IA tendremos presente prioritariamente al investigador implicado, de quien cuestionaremos el posicionamiento y, por consiguiente, la postura. Un mayor o menor grado de intervención es posible: de la observación externa del investigador a la observación participante, por ejemplo, en la cual el investigador puede inducir eventos y hechos sobresalientes que modifican el dispositivo. El quehacer del investigador, su posicionamiento teórico y sus opciones de investigación inducen entonces ampliamente a la metodología en cuestión.

Cierto número de parámetros entran en relación en la IA, lo cual Wallace describe en estos términos:

[...] le but fondamental, le champ d'action, le centre d'intérêt premier de ceux qui conduisent la recherche, la conception de la manière de conduire la recherche, l'arrière-plan éthique qui préside au contrôle des résultats de la recherche, la manière dont les sciences sociales s'impliquent pour relier théorie et pratique les méthodes de recherche les moyens que l'on se donne pour établir les critères de validité de la recherche, les moyens dont disposent ceux qui conduisent la recherche, etc. $(97)^{5}$

Cabe anotar que se subrayan las maneras, métodos y medios que favorecen la conducción de la investigación, más que los objetivos de esta.

\footnotetext{
Nuestra traducción: "El objetivo fundamental, el campo de acción, el centro de interés primero de los que conducen la investigación, la concepción de la manera de conducir la investigación, el segundo plano ético que preside al seguimiento de los resultados de la investigación, la manera cómo las ciencias sociales se implican para relacionar teoría y práctica, los métodos de investigación, los medios que se dan para establecer los criterios de validez de la investigación, los medios de los cuales disponen los que conducen la investigación, etc." (Wallace, 97).
}

La cientificidad de los resultados, su robustez —esto depende de la metodología utilizada - son, a menudo, cuestionados en la IA. Sin duda, el resurgimiento de la ia al final de la década del sesenta, en Francia, no es completamente extranjero al desconocimiento, casi al desinterés que suscita en los círculos científicos, pues ha sido asociado con los excesos de participación de ciertos grupos innovadores que se autoadministran y a la imprecisión organizacional de la época, producto de numerosas inversiones de los valores.

Sin embargo, estas representaciones no han sido menospreciadas por las tentativas de conceptualización más recientes, posteriores al resurgimiento de la metodología de la ia en la década del ochenta (Aguado; Goyette y Lessard-Hebert; Grotjahn), ni por los intentos de clarificación de su metodología (Thirion; Liu; Resweber; Van den Maren).

La IA tiende tanto hacia el polo científico como al praxeológico. Es una metodología, por decirlo de alguna manera, mixta. En este sentido, sufre, sin lugar a dudas, un déficit de clarificación terminológica y de una ambigüedad en cuanto a la articulación entre los dos términos "investigación" y "acción". Asimismo, este pequeño guion entre investigación y acción crea bastantes problemas, los cuales destaca la pluma de Henri Portine:

Une recherche-action suppose une dialectique entre recherche et action : la recherche fait progresser l'action et l'action fait progresser la recherche. Une recherche qui vise l'action n'est pas une rechercheaction. C'est une recherche qui prend en compte le coût social et l'intérêt social de cette recherche. Ce qui est somme toute fort honorable $(267)^{6}$

Estar en IA significa estar en situación, siendo esta última principalmente operada por los actores. La situación, tal como lo señala Bazin, en el mismo sentido que Barbier o Ellis, es la unidad básica de la IA, pues esta es descrita en su ambiente. Las ciencias sociales ofrecen un fuerte potencial de libertad y de creatividad para los investigadores (ver Bazin), algo que no sucede siempre con la DDLC.

\footnotetext{
6 Nuestra traducción: "Una investigación-acción supone una dialéctica entre investigación y acción: la investigación hace progresar la acción y la acción hace progresar la investigación. Una investigación que tiene como objetivo la acción no es una investigación-acción. Es una investigación que tiene en cuenta el costo social y el interés social de esta investigación. Algo que es, después de todo, muy honorable" (Portine 267).
} 
Si es importante trabajar en situación abierta, es porque hay que respetar al máximo - de los actores implicados-, la posibilidad de definir lo que hacen, construir su propia categoría semántica de cualificación y no la que es impuesta por externos. Una situación nunca admite un solo punto de vista, una sola forma de comprenderla; está abierta a otras formas de comprensión que representan diferentes perspectivas de evolución (Bazin).

Podría decirse que la mirada puesta sobre una situación está acompañada de un análisis, luego de una interpretación de los fenómenos (hechos o prácticas) observados (Van den Maren), y esto se da a la luz de las teorías de referencia del campo y de una axiología.

La IA está fuertemente unida a sus contextos de intervención y se inscribe en un doble campo. Por una parte, un campo exploratorio, descriptivo y explicativo; $y$, por otra, un campo operativo, comprensivo y con intención de cambio, según Macaire, en "Monisme ou pluralisme?". Tal postura de investigación es implicada y pragmática a la vez.

Pasaremos por tres etapas sucesivas: la primera concierne a la búsqueda del conocimiento, que es el objeto de la investigación; la siguiente tiene que ver con la reflexión epistemológica y ética en investigación; $\mathrm{y}$, finalmente, la última concierne al paso de un posicionamiento de investigador a una postura de investigación.

\section{La doxa, desventaja para el acceso al conocimiento}

La investigación en DDLC plantea como objeto de estudio el conocimiento de los aprendices, de sus estrategias de aprendizaje, de sus relaciones con las lenguas - entre ellos y con sus docentes, así como con los recursos que utilizan-, y de los resultados de sus aprendizajes en contextos precisos.

En el mito de la caverna, el filósofo Platón en $\mathrm{La}$ república, presenta el conocimiento como un fenómeno difícilmente accesible, pues el ojo es engañado por sombras perturbadoras que la mente humana toma como realidades. Todo empieza 400 años antes de Jesucristo, en una gruta donde los hombres están encadenados:

Ils tournent le dos à louverture et ne peuvent voir la lumière quen regardant son reflet sur les parois de la caverne. Ces hommes se trouvent dans cette position depuis leur enfance et ils nont rien connu d'autre.
Personne n'est venu les rencontrer, personne ne leur a expliqué comment cétait dehors. Pour eux, la vérité, ce sont les ombres qu'ils aperçoivent sur les murs. Ils pensent que lorsqu'ils voient l'ombre d'un animal qui passe devant la grotte, cette ombre est le vrai animal. Un jour, un homme est libéré. Malgré sa grande peur, il décide de sortir de la caverne pour découvrir ce qu'il y a à l'extérieur. Ses yeux lui font d'abord très mal parce que le soleil l'éblouit, mais peu à peu il commence à s'y habituer. Au bout d'un moment, il distingue les formes, puis les détails de ces formes et enfin les couleurs. Quand un lion passe près de lui, il le reconnait à sa taille mais voit aussi la couleur de sa crinière, le tranchant de ses dents et arrive même à toucher la douceur de son poil. Après avoir passé plusieurs heures à observer les moindres détails de ce nouveau monde, il pense que ses amis enchainés ont eux aussi le droit de savoir. Il les rejoint et leur raconte tout : les arbres, les animaux, le vent, les couleurs, le soleil. Il leur explique même que ce qu'ils ont cru depuis toujours n'est pas la vérité mais une simple ombre de la vérité. Malheureusement pour lui, personne ne veut le croire : tous pensent qu'il raconte n'importe quoi, qu'il devient fou. Certains proposent même de le tuer. (http://mercigeorges.com/2009/02/) ${ }^{7}$

Los primeros obstáculos para acceder al conocimiento provienen de la experiencia misma del humano. Las sombras de los animales no son lo real, son obstáculos para el conocimiento de los animales fuera de la caverna; impiden saber qué es un animal y creerle a

\footnotetext{
7 Nuestra traducción: "Dan la espalda a la entrada y sólo pueden ver la luz que se refleja en las paredes de la caverna. Estos hombres se encuentran en esta posición desde su infancia y no conocen nada más. Nadie ha venido a su encuentro, nadie les ha explicado cómo es afuera. Para ellos, la verdad son las sombras que perciben sobre los muros. Piensan que, al ver la sombra de un animal que pasa frente a la caverna, esta sombra es el verdadero animal. Un día, un hombre es liberado. A pesar de su gran miedo, decide salir de la caverna para descubrir lo que hay en el exterior. Sus ojos le duelen mucho al principio debido al sol que los deslumbra, pero poco a poco comienza a habituarse. Luego de un momento, distingue las formas, luego los detalles de estas formas y finalmente los colores. Cuando un león pasa cerca de él, reconoce su tamaño pero ve también el color de su crin, lo afilado de sus dientes e incluso toca la suavidad de su pelaje. Luego de haber pasado varias horas observando los mínimos detalles de este nuevo mundo, piensa que sus amigos encadenados tienen también derecho a saber. Los reúne y les cuenta todo: los árboles, los animales, el viento, los colores, el sol. Aún más, les explica que lo que ellos creen desde siempre no es la verdad sino una simple sombra de la verdad. Infortunadamente para él, nadie quiere creerle: todos piensan que está desvariando, que se ha vuelto loco. Aún más, algunos proponen matarlo. (http:// mercigeorges.com/2009/02/)
} 
quien tiene una experiencia diferente con los animales, por ejemplo. Las creencias son doblemente peligrosas, para el saber y las relaciones con las demás experiencias. Estas imágenes constituyen representaciones individuales pero también sociales. Según Bachelard, son constructos, obstáculos epistemológicos y no reales.

Todos los humanos no tienen la misma experiencia, por consiguiente no tienen el mismo acceso al conocimiento. Estas sombras y estas opiniones, la doxa, son creencias estereotipadas que conviene destruir o superar a fin de acceder al conocimiento. La búsqueda del conocimiento pasa por la superación de la doxa y la objetivación de los saberes sobre lo real. El conocimiento, que es en sí mismo un constructo, se organiza progresivamente por apilamiento y deconstrucción de saberes provisionales. Tal actitud supone tomar un riesgo, una incomodidad, ya que son cuestionadas las convicciones y certezas anteriores.

\section{Reflexión epistemológica y ética en el corazón de la investigación en DDLC}

Adoptar una conducta científica es, ante todo, renunciar a la doxa y cuestionarse. La IA es particularmente favorable a la emergencia de situaciones problema, en las cuales los cuestionamientos son vivos y la construcción progresiva gracias a los saberes preconstruidos.

\section{De la evidencia de los hechos a las teorías de referencia}

Las representaciones de los hombres son el reflejo de lo que consideran es "verdadero". Es en el corazón de las creencias que se debe buscar eso en lo que creen o quieren creer los humanos. La realidad sólo existe a través de este filtro, tal como escribe Bernard Lahire:

Jamais les faits n'imposent leur évidence; ils supposent toujours un regard (ou un point de vue) qui les constitue. [...] Une chose est sûre: aucune enquête de terrain, ni aucune base de données, quels que soient son étendue et son degré de précision, n’ont jamais engendré et n'engendreront jamais par elles-mêmes des connaissances, si elles ne sont pas conçues, guidées, suscitées, informées, alimentées par une imagination théorique. $(13)^{8}$

8 Nuestra traducción: "Los hechos no imponen nunca su evidencia; suponen siempre una mirada (o un punto de vista) que los constituye. [...] Una cosa es segura: ninguna base de datos ni
Ahora bien, esta imaginación teórica está sujeta a los registros culturales por los que el individuo ha atravesado, más o menos de forma consciente. Lahire habla de matizadores culturales individuales disonantes, estadísticamente más frecuentes actualmente que en la época de la caverna de Platón, y más frecuentes que los "matizadores concordantes", culturalmente en acuerdo. Las influencias de la sociedad y de las experiencias vividas por los individuos forjan su punto de vista y contribuyen a cambiar su visión de la realidad, en todo caso a diversificar sus puntos de vista. Ya no estamos en un mundo homogéneo y unívoco, si acaso algún día lo fue. Vivimos en un mundo complejo, en el que los individuos tienen posiciones a veces contradictorias con sus mismos actos. De allí cierta dificultad para ir hacia teorías de referencia claras en el mundo de las ciencias humanas y sociales.

La imaginación teórica de los individuos está vinculada a un punto de vista sobre el mundo, a una visión de la realidad, a una concepción de la verdad. Cualquier investigación refleja convicciones y elecciones científicas, es decir, conceptos retenidos de manera consciente como válidos. Es el lenguaje que vehicula las teorías subyacentes y, muy a menudo, inconscientes. El investigador -en IA particularmente - genera pensamientos construidos por la acción, objetiva sus constataciones y hace corresponder lo que piensa y afirma con las teorías de referencia que le son propias; además, establece cruces científicos. Ahora bien, cualquier construcción de saber supone conocimientos teóricos y referentes que sirven de vínculo entre teorías. No se trata de "hacer un poco de todo", sino de objetivar constructos científicos sólidos que tengan en cuenta a la vez los constructos sociales y los conocimientos humanos.

En este campo, la reflexión y la prudencia ontológica son apropiadas. Narcy-Combes considera que:

Le recul épistémique soulève un grand nombre de questions: l'approche scientifique d'un problème impose de déterminer quels sont les obstacles épistémologiques en jeu, de valider ou non les « autorités » et les observations, de repérer les biais partagés de la communauté de pratique à laquelle on appartient et de mesurer les effets de contexte. (119) ${ }^{9}$

ningún trabajo de campo, sin importar su extensión ni grado de precisión, ha generado alguna vez ni generará por sí solo conocimientos, a no ser de que sea concebido, guiado, suscitado, informado o alimentado por una imaginación teórica” (Lahire 13).

9 Nuestra traducción: "La distancia epistémica suscita un 
Observando los términos utilizados para caracterizarla, puede constatarse que la IA se inscribe en el campo de las teorías de la acción y las del cambio. El recurso a la teoría permite la objetivación de las intuiciones que surgen de la observación y de la experiencia implicada, la cual favorece el distanciamiento crítico y la reflexión distanciada. Para el investigador que está a menudo en el corazón de la acción, la explicitación es una herramienta que le ayuda a pensar y a construir. Las teorías científicas de referencia sobre las cuales se apoya el investigador son a la vez balaustradas, referentes y una caución científica para su investigación. Estas teorías de referencia son externas al investigador, objetivables y le sirven de sustento.

La apuesta es la misma: buscar reconocimiento para este tipo de investigación. Es posible seguir en este sentido la posición de Kuhn, para quien solo las investigaciones fuertemente arraigadas en la tradición científica contemporánea tienen oportunidad de romper esta tradición y dar nacimiento a una nueva (307).

La IA no es solamente un marco de intervención, algo que remite a la metodología utilizada e insiste sobre los andamiajes teóricos; es también un espacio de libertad, tal como lo sugiere Barbier. Con respecto a lo anterior, la IA se apoya en la libertad del investigador. El paso del marco a un espacio abierto tiene que ver con una elección epistemológica, la cual se realiza por referencia a las posiciones teóricas que alimentan la reflexión, a un enfoque holístico.

En la medida en que la IA se ancle en lo real de la acción, podría pensarse que se limita a ello. Ahora bien, no es este el caso. Esta se interesa por las situaciones que tienen como objetivo o que operan el cambio, por la elaboración de nuevos conocimientos sobre las lenguas, sobre los aprendices y sus sistemas de representación o sus estrategias de aprendizaje, etc. Al afirmar esto, sostenemos que la IA tiene como objetivo, a su vez, conductas de acción, conductas de investigación y de transformación del objeto. Excluye los enfoques que solo tienen como intención la acción o la investigación. Esto resulta justamente esencial y a la vez está siempre cargado de un potencial de incomodidad y, aún más, de ambigüedad.

sinnúmero de preguntas: el enfoque científico de un problema impone determinar cuáles son los obstáculos epistemológicos en juego, validar o no las 'autoridades' y las observaciones, señalar los sesgos compartidos de la comunidad de práctica a la cual se pertenece y medir los efectos de contexto" (Narcy-Combes, 119).

\section{El ethos}

El ethos remite a los valores que fundan la acción. Esos valores constituyen un conjunto individual que hace referencia a las culturas atravesadas y a menudo hibridadas. Para Descartes, en 1673, la ética es reflexión y juicio y precede a la acción; el filósofo de la Ilustración se apoya en la primacía de la razón a fin de acceder al conocimiento.

La racionalidad cartesiana proponía tres planos en el acceso al saber: 1) el plano pragmático, el de la acción; 2) el plano epistemológico, este es el de las teorías de referencia explicativas en investigación; y 3) el plano ético, el de los valores. Ahora bien, todo confluye, pasando sin cesar de uno al otro. Es imposible separar los tres planos (Le Moigne). El triángulo solo existe para fines de clarificación y análisis.

En la doble relación del conocimiento (que esclarecen las teorías de referencia) y de la acción (que plantean los problemas y creencias encontradas), el ethos ofrece el medio para movilizar la inteligencia. Solicita nuevas herramientas intelectuales.

Más que oponer monismo (en búsqueda de objetivación, de leyes y dogmas), a pluralismo (más ligado al compromiso del sujeto), privilegiamos una concepción más comprensiva de la IA en DDLC, la cual toma prestadas de las dos metodologías sus fuerzas respectivas. El discurso de la IA se presenta más como un espacio dialéctico y argumentativo, compuesto por una diversidad de razonamientos metodológicos (a veces contradictorios), los cuales entran en confrontación con el fin de dar cuenta de una realidad compleja y resaltar la multiplicidad de los puntos de vista (Macaire, "Monisme ou pluralisme ?" 75).

\section{Del posicionamiento del investigador a una postura de investigación}

La ambigüedad es, a menudo, mantenida por los investigadores y practicantes mismos. El investigador y el practicante son actores de la IA. Están ambos, muy a menudo, comprometidos en una conducta de acción, casi de intervención. Es, sin embargo, muy importante conservar una distinción entre ellos. El investigador se caracteriza por la búsqueda de su objeto de investigación en el respeto por la cientificidad y la ética que lo anima; el practicante por su experimentación, su cuestionamiento reflexivo y la búsqueda de soluciones, aun cuando ambos están implicados. 
Cuando el investigador se refiere a sus teorías de referencia, es consciente de su posicionamiento científico, de los conceptos integradores de su investigación y de los referentes a los cuales apela. Está, por ende, en coherencia con ellos. Así como en el campo de las ciencias exactas, la IA en DDLC permite clarificar relaciones entre las teorías convocadas.

Ahora bien, el quehacer del investigador no es únicamente convocar referentes teóricos comprobados. En la medida en que él deba igualmente producir conocimiento, debe también adoptar una postura de investigación. El investigador en IA adopta una triple posición para:

- Cuestionar: considerar los saberes constituidos como ayudas para la comprensión y no solamente como obstáculos.

- Explicitar: concientizar el “ipor qué?”; dar cuenta de él e ir hacia el "ipara qué?”, según el modelo de la práctica de la reflexividad de Schön, el cual permite conocer el ambiente y el contexto conociéndose a sí mismo.

- Correlacionar: aceptar el juego de las circulaciones en el marco de un intervencionismo científico.

Por otra parte, se remite aquí al análisis hecho de esas tres dimensiones de una postura que supera el posicionamiento (Macaire, "Monisme ou pluralisme ?"; Narcy-Combes). La relación entre implicación (práctica, en la acción) y distanciamiento (teórico), caracteriza la diferencia entre ellas. Esto se hace evidente en las herramientas conceptuales de las cuales se dota el investigador (problemática, hipótesis, instrumentos de experimentación, teorías de referencia, etc.); los instrumentos de recolección de datos y de nativización que convoca (Demaizière y Narcy-Combes); y su capacidad para hacer que su investigación sea extrapolable y, por ende, tenga un grado de robustez calificado (Seliger \& Shohamy; Schlemminger).

En estas condiciones, la IA puede vanagloriarse de tener un estatus científico, ya que es exigente de cara a sí misma y exige una verdadera postura de investigador.

Por postura, se entiende aquí, tal como lo señala Rémi Hess:

Le système d'attitudes et de regards vis-à-vis des partenaires, des situations, des objets, dans le cadre des recherches ou des pratiques sociales. [...] Une telle posture dépend au moins autant des caractéristiques de la situation où elle vient s'inscrire, et des représentations que s'en donnent nos partenaires, que de notre intentionnalité, de nos stratégies et de nos procédures. $(9)^{10}$

\section{Conclusión}

La diversidad y la riqueza de estos parámetros muestran hasta qué punto el fenómeno es complejo y merece alguna reflexión, sin descuidar la relación entre estos dos parámetros. En este artículo se aceptan como indicadores de éxito de una IA llevada a cabo científicamente - además de los tres campos esbozados más arriba-, el análisis de la situación de partida, la importancia del grupo social de actores, el trabajo sobre las prácticas reflexivas regulares, los incidentes críticos y los hechos sobresalientes; de igual forma, los productos y las marcas escritas cruzadas, así como las posibilidades de transferencia a otras situaciones como constitutivos indicadores de realización científica de una IA en DDLC.

La dificultad a la cual se confronta el investigador en IA es la de la tensión entre "implicación" y "distancia". Goza de un rol de intermediario - el de investigador-acompañante-, y de un espacio en tensiones, un marco provisional siempre renovado de organización de saberes. Es su responsabilidad mantener la vigilancia ética, epistemológica y metodológica, fundamento de su postura responsable. Para esto, dispone de herramientas conceptuales y metodológicas.

La investigación-acción vive una multiplicidad de formas de acción y de investigación, a un tiempo, entre las dos palabras que vincula el guion. Es a la vez su debilidad, un riesgo y su fuerza, si se acepta vivir en la complejidad para estar en el corazón de lo que entra en juego en la caverna o en el corazón de la enseñanza/aprendizaje de las lenguas.

Si se acuerda decir, en la misma línea de Kuhn, que la investigación funciona no por continuidad y acumulación, sino por rupturas, es decir, de manera discontinua, los nuevos objetos de la investigación exigen, por ende, nuevas formas de enfoques comprehensivos y de tratamiento en términos de combinatorias. Dirigirse hacia una ia responsable que comprometa una postura de investigación asumida y un posicionamiento científico, el cual renuncie

10 Nuestra traducción: "El sistema de actitudes y de miradas de cara a los compañeros, situaciones, objetos, en el marco de investigación o de prácticas sociales. [...] tal postura depende al menos tanto de las características de la situación en la cual se inscribe y las representaciones que los compañeros se hacen de la misma, como de nuestra intencionalidad, de nuestras estrategias y nuestros procedimientos" (9). 
al bricolaje científico, es dirigirse hacia una ética profesional.

\section{Referencias}

Aguado, Karin (ed.). Zur Methodologie in der empirischen. Fremdsprachenforschung, Hohengehren: Schneider Verlag, 2000. Impreso.

Bachelard, Gaston. La formation de l'esprit scientifique. Paris: Vrin, 1988. Impreso.

Barbier, René. La recherche-action. Paris: Anthropos, 1996. Impreso.

Bazin, Hugues. Méthodologie en recherche-action. 2004. Web.

CNRs. Schéma stratégique. CNRs: Paris, 2002. Impreso.

Demaiziere, Françoise y Jean-Paul Narcy-Combes. "Méthodologie de la recherche didactique: nativisation, tâches et TIC". Apprentissage des langues et systèmes d'information et de communication (ALSIC), 8.1 (2005): 45-64. Web. DoI: $10.4000 /$ alsic.326

Goyette, Gabriel y Michelle Lessard-Hebert. La recherche action: ses fonctions, ses fondements, son instrumentation. Québec: Presses Universitaires du Québec, 1988. Impreso.

Grotjahn, Rüdiger. "Empirische Forschungsmethoden: Überblick". Eds. Bausch, Karl-Richard, Christ, Herbert, Krumm y Hans-Jürgen. Handbuch Fremdsprachenunterricht. Tübingen: Francke Verlag, 1995. 457-461. Impreso.

Habermas, Jürgen. Théorie de l'agir communicationnel. Paris: Fayard, 1987. Impreso.

Hess, Rémi. "Recherche-action et formation, le travail de terrain”. Pratiques de formation/Analyses, 18 (1989). Impreso.

Kuhn, Thomas. La tension essentielle. Paris, Gallimard, 1977. Impreso.

Lahire, Bernard. La culture des individus. Dissonances culturelles et distinction de soi. Paris: La Découverte, 2004. Impreso.

Lemoigne, Jean-Louis. "Les enjeux éthiques de la didactique des langues et des cultures nappellent-ils pas un nouveau discours sur la méthode des études de notre temps? ". ELA, 140 (2005): 421-433. Impreso.
Lewin, Kurt. "Forces behind Food Habits and Methods of Change". Bulletin of National Resources Council, 108 (1943): 35-65. Impreso.

Liu, Michel. Fondements et pratiques de la recherche-action, Paris: L'Harmattan, 1977. Impreso.

Macaire, Dominique. "Didactique des langues et recherche action”. Les Cahiers de l'Acedle, NeQ, 4 (2007): 93-120. Web.

----. "De la didactique de l'allemand à une didactique du plurilinguisme, la recherche-action comme aide au changement". HDR, 1 (2009). http://acedle.org/IMG/ pdf/HDR_Macaire_SyntheIEURse_2008.pdf

----. "Monisme ou pluralisme? Vers une conception compréhensive de la recherche-action en didactique des langues et des cultures". Le Français dans le Monde, Recherches \& Applications, 48, (2010): 66-75. Impreso.

Narcy-Combes, Jean-Paul. Didactique des langues et TIC: Vers une recherche action responsable. París: Ophrys, 2005. Impreso.

----. "Recherche-action et illusion ontologique en didactique des langues". Le Français dans le Monde, Recherches \& Applications, 48 (2010): 111-123. Impreso.

Portine, Henri. "Analyse de la thèse de J. Rézeau". ALSIC, 5.2 (2002): 259-268. Impreso.

Resweber, Jean-Paul. La recherche-action. París: PUf. 1998. Impreso.

Schlemminger, Gerald. "Methoden in der Fremdsprachenforschung-ein kurzer Überblick”. Karlsruher Pädagogische Beiträge, 57 (2004): 20-34.

Schön, Donald. Le praticien réflexif. Montréal: Editions logiques, 1994. Impreso.

Seliger, Herbert W. y Elana Shohamy. Second Language Research Methods. Oxford: oup, 1989. Impreso.

Thirion, Anne-Marie. Tendances actuelles de la rechercheaction. Examen critique. Thèse de doctorat en Sciences de léducation, Université de Liège, 1980. Impreso.

Van Der Maren, Jean-Marie. La recherche appliquée en pédagogie. Des modèles pour lenseignement. ParisBruxelles : De Boeck Université, 1999. Impreso. doi: 10.3917/dbu.maren.2003.01

Wallace, Mike. "A historical Review of Action Research". Journal of Education for Teaching, Routledge, 13.2 (1987): 97-119. doi: 10.1080/0260747870130201 